\title{
Análisis médico forense del peso pulmonar y de otros factores en la muerte por sumersión
}

\section{Medical forensic analysis of the pulmonary weight and of other factors in the death for drowning}

\begin{abstract}
Resumen
La investigación médico-legal de la muerte por sumersión es uno de los temas más controvertidos y difíciles en medicina forense. Se han propuesto diversos hallazgos durante la autopsia, pruebas histopatológicas y biológicas, pero ninguna de ellas es específica ni definitiva en el diagnóstico de este tipo de muerte. En este artículo analizamos 78 casos de fallecidos en los que se estableció un diagnóstico médico-legal de muerte por sumersión. El 18\% tenían pulmones con un peso inferior a $1.000 \mathrm{~g}$. En el 3,8\% los pulmones eran macroscópicamente normales, los conocidos como pulmones secos. En algunos de los casos considerados en la literatura médica como ahogados secos la muerte puede deberse a causas naturales de difícil diagnóstico.
\end{abstract}

Palabras clave: Sumersión. Peso pulmón. Pulmón seco. Patología forense.

\begin{abstract}
The medico-legal investigation of death by drowning is one of the most controversial and difficult issues in forensics. Have been proposed several findings at autopsy, histopathological and biological tests but none of them are specific and definitive diagnosis of this type of death. We analyze 78 cases of deaths in which a forensic diagnosis of death by drowning was established. In $18 \%$ of the total had lungs with a weight less than $1,000 \mathrm{~g}$. $3.8 \%$ the lungs were normal macroscopic, known as dry lungs. Some of the cases in the medical literature as dry drowning death may be due to natural causes of difficult diagnosis.
\end{abstract}

Key words: Drowning. Lung weigth. Dry lung. Forensic pathology.

\section{Introducción}

Uno de los temas más difíciles de la patología forense es la muerte por sumersión; de hecho, muchos tratados prefieren hablar de cadáveres rescatados del agua. Simonin la definió como un mecanismo de muerte originado por respirar debajo del agua o por perder la respiración debajo del agua ${ }^{1}$. Debido a la gran variedad de definiciones existentes en la literatura científica, fue en el I Congreso Mundial de Ahogamiento celebrado en Amsterdam, en el año $2002^{2}$, donde se consensuó el concepto de "sumersión", siendo este el proceso de experimentar insuficiencia respiratoria por la sumersión o inmersión en un líquido; después de este proceso, la víctima puede fallecer o sobrevivir, pero independientemente del resultado final, la víctima sufrió un episodio de sumersión. Asimismo se llegó a un consenso para abandonar el concepto de near drowning o casi ahogamiento (supervivencia inmediata que sigue a la asfixia debido a la sumersión en un medio líquido), por la confusión que origina en la literatura médica de carácter clínico.

Según la Organización Mundial de la Salud ${ }^{3}$ y el European Resucitation Council se producen 450.000 muertes al año por ahogamiento y se calculan 1,3 millones de años de vida perdidos. En Europa, por cada muerto se calculan de uno a cuatro accidentes graves con hospitalización. Es la tercera causa de muerte accidental en los Estados Unidos (8.000 muertes/ año), y de ellos se producen un $50 \%$ en piscinas, un $20 \%$ en el mar y un $15 \%$ en el hogar (bañeras); es

\section{F. Martín Cazorla ${ }^{1}$ \\ L. Rubio Lamia² \\ V. Ramos Medina ${ }^{3}$ MJ. Gaitán Arroyo ${ }^{4}$ \\ IM. Santos Amaya ${ }^{5}$}

${ }^{1}$ Médico Forense. Servicio de Patología Forense del IML de Málaga.

2Profesora Doctora Ayudante. Área de Medicina Legal y Forense. Universidad de Málaga.

${ }^{3}$ Médico Forense. Jefe de Sección de Histopatología y Anatomía Forense. Servicio de Patología Forense del IML de Málaga.

${ }^{4}$ Doctora en Biología. Área de Medicina Legal y Forense. Universidad de Málaga. ${ }^{5}$ Profesor Titular. Área de Medicina Legal y Forense. Universidad de Málaga.

\section{Correspondencia:}

Fernando Martín Cazorla

Servicio de Patología Forense. Instituto de Medicina Legal de Málaga.

Ciudad de la Justicia.

C/ Fiscal Luis Portero García. 29010 Málaga

E-mail: fernando.martin.ius@ juntadeandalucia.es

Fecha de recepción: 30. MAR. 2014

Fecha de aceptación: 26. JUN. 2014 
la segunda causa de muerte en menores de 30 años y la primera en los niños ${ }^{4}$. Según datos del Instituto Nacional de Estadística ${ }^{5}$, en el año 2012 en España fallecieron por sumersión 438 personas, de las cuales 358 eran hombres y 80 mujeres; la mayoría de las muertes tuvo lugar en mayores de 20 años.

La investigación médico-legal de la muerte por sumersión no siempre es fácil para el médico forense. Cuando un cadáver aparece sumergido en el agua se plantean muchas cuestiones que el médico forense debe estar en condiciones de poder responder o, al menos, acercarse lo máximo posible a su respuesta. Como señala Knight ${ }^{6}$, el diagnóstico médico forense de la muerte por sumersión es uno de los mayores problemas en patología forense, en especial cuando los fenómenos putrefactivos aparecen en el cadáver. Así, la víctima puede haber fallecido de forma natural o violenta antes de caer al agua, mientras está en el agua o debido a los efectos de padecer un episodio de sumersión.

Los hallazgos durante la autopsia y las pruebas complementarias por sí solas no pueden establecer un diagnóstico de certeza de muerte por sumersión, ya que no existe ningún dato específico de este tipo de muertes. Por ello, deberemos apoyarnos en el tercer pilar básico de la investigación médico-legal de la muerte, cual es el levantamiento del cadáver. Sólo con estos datos analizados de forma conjunta podemos aproximarnos de manera definitiva al diagnóstico médico-legal de muerte por sumersión.

No es el objetivo de este artículo realizar un estudio pormenorizado del mecanismo fisiopatológico de la muerte por sumersión ni de los diferentes hallazgos necrópsicos, histopatológicos o biológicos; para ello remitimos al lector a múltiples obras y artículos sobre el tema, y como excelente referencia a la revisión realizada por el Dr. Romero Palanco ${ }^{7}$.

Se ha citado como un signo esencial de la muerte por sumersión la presencia de edema pulmonar y enfisema acuoso, consecuencia de la abrupta entrada del medio de sumersión en las vías respiratorias, con la consiguiente afectación de alvéolos y capilares, pasando parte del líquido de sumersión y del aire al espacio intersticial. Esto origina los conocidos como "pulmones húmedos", que representan del 80-90\% de los casos según las series revisadas. Sin embargo, hemos de indicar que este hallazgo es totalmente inespecífico y podemos encontrarlo en sujetos fallecidos por otras causas (muertes relacionadas con drogas de abuso o patología cardiaca). Esto permite afirmar a Shkrum y Ramsay ${ }^{8}$ que, en adultos mayores de 18 años, un peso de los pulmones superior a 1.000 g sería significativo a efectos de distinguir entre muerte por sumersión y otras causas de muerte.

Por otra parte, un $10-15 \%$ de los cuerpos recuperados del agua no tienen líquido en los pulmones. Diferentes autores sugieren el espasmo de glotis como el mecanismo que impide la entrada de agua en las vías respiratorias. La apnea inicial podría estimular a los quimiorreceptores carotídeos, contribuyendo al desencadenamiento de una parada cardiaca de origen vagal, la cual podría ser igualmente facilitada al entrar en contacto con el agua?.

Es por este motivo por lo que, cuando en la autopsia la presencia de líquido en los pulmones es insignificante, se habla de muertes en el agua con pulmones secos o ahogamiento seco (dry-drowning) ${ }^{9}$. Algunos autores ${ }^{10}$ consideran que el volumen de líquido aspirado varía de unas víctimas a otras, dependiendo de factores como la frecuencia y la duración del laringoespasmo, el número y la profundidad de los movimientos realizados antes de la muerte, y el tiempo transcurrido hasta la parada cardiaca. Para estos autores, los pulmones secos pueden tener varias explicaciones además del laringoespasmo, como la inhibición cardíaca vagal desencadenada por el contacto del agua con las vías respiratorias superiores, la parada cardíaca súbita o algunos reflejos pulmonares ${ }^{10}$.

En la actualidad existe poco conocimiento sobre los factores que caracterizan a los pulmones con bajo peso y los mecanismos involucrados ${ }^{9-12}$. Incluso se cuestiona su frecuencia, y diversos autores indican que su incidencia real es más baja de lo que se venía considerando hasta ahora, situándose alrededor del $2 \%$. Además, estiman que los cuerpos humanos encontrados en el agua con pulmones aparentemente normales, desde un punto de vista macroscópico e histopatológico, podrían encubrir más casos de muertes súbitas de origen natural en el agua de los que habitualmente se conocen y, por tanto, se clasificarían erróneamente como muertes por sumersión; nos estamos refiriendo a los casos de canalopatías con expresión cardíaca (síndrome de QT largo, síndrome de Brugada, taquicardia ventricular paroxística). Es un hecho conocido que, en estos sujetos, el baño puede ser un factor desencadenante de arritmias.

Es en este punto donde la conocida como autopsia molecular tiene una aplicación innegable en la patología forense. Estas técnicas deben ir incorporándose de manera creciente al campo de la patología forense y contar con la colaboración de laboratorios de genética.

El objetivo de este artículo es analizar los factores individuales, los hallazgos macroscópicos en la au- 
topsia y las pruebas complementarias realizadas en víctimas fallecidas por sumersión en la provincia de Málaga entre los años 2004 y 2011. Se ha prestado especial atención al análisis correlacional según el peso de los pulmones.

\section{Material y métodos}

En este estudio se incluyeron los casos de muerte por sumersión acontecidos en la provincia de Málaga entre los años 2004 y 2011 a los que se practicó la autopsia en el Servicio de Patología Forense del Instituto de Medicina Legal de Málaga. El número total de casos durante este periodo fue de 200. Las autopsias se realizaron siguiendo la Recomendación No 99(3) del Consejo de Ministros de los Estados Miembros, para la armonización metodológica de las autopsias médico-legales ${ }^{13}$.

Dentro del grupo de cadáveres encontrados en el agua, para el análisis del peso pulmonar y para evitar falsos positivos se establecieron los siguientes criterios de exclusión:

- Edad inferior a 18 años.

- Inicio de los procesos putrefactivos.

- Intervalo post mórtem superior a 6 días.

- Práctica de reanimación cardiopulmonar tras la extracción del agua.

Como consecuencia de los criterios anteriores se obtuvo un tamaño muestral definitivo de 78 casos.

Se elaboró una base de datos mediante el programa Excel (Microsoft ${ }^{\circledR}$ ) en la cual se analizaban las siguientes variables de cada caso:

- Factores individuales tales como sexo, edad, líquido de sumersión (agua salada o dulce), intervalo post mórtem, peso del corazón, existencia de patología cardíaca o pulmonar y tóxicos en sangre u orina.

- Factores circunstanciales, incluyendo testimonios fehacientes de la sumersión de la víctima, notas de despedida, estación del año y etiología médico-legal de la muerte.

- Hallazgos macroscópicos durante la autopsia: peso de los pulmones, presencia de hongo de espuma, espuma traqueobronquial, derrame pleural, equimosis de Paltauf o agua en el estómago.

- Hallazgos histopatológicos: enfisema acuoso, hemorragia alveolar, rotura de septos alveolares, edema pulmonar y disminución del número de macrófagos alveolares.

- Estudio biológico de diatomeas en la médula ósea y en el bazo.

Los datos se analizaron utilizando el programa estadístico GraphPad Prism v.5 (GraphPad Software Inc., La Jolla, CA, USA). Los resultados se describen como porcentaje de la media \pm desviación estándar. Todas las variables descritas fueron correlacionadas con el peso de los pulmones. Así, los datos obtenidos de los 78 casos de sumersión se analizaron mediante el coeficiente de correlación de Pearson y regresión logística multivariada para la dependencia lineal, utilizando el peso del pulmón como la variable dependiente. Se consideró significativo un valor de $p<0,05$.

\section{Resultados}

Entre los años 2004 y 2011, ambos inclusive, se realizaron 200 autopsias de víctimas de sumersión en el Servicio de Patología Forense del Instituto de Medicina Legal de Málaga. Tras la aplicación de los criterios de exclusión la muestra final fue de 78 casos, de los cuales 56 eran hombres, con una edad media de 57,79 $\pm 17,85$ (rango: 20-89), y 22 eran mujeres con una edad media mayor que la de los hombres (68,45 $\pm 12,6$; rango: $49-87)$. El 70,5\% de los casos fue verificado por la presencia de testigos que vieron lo ocurrido o por la existencia de notas de despedida en el caso de los suicidios.

Respecto a la etiología médico-legal de la muerte, la accidental fue la más frecuente, con 54 casos, seguida de la suicida con 22 casos y la homicida con 2 casos. En los hombres, la etiología médico-legal accidental fue la más frecuente $(83,9 \%)$, mientras que en las mujeres lo fue la suicida $(63,7 \%)$.

En el $52,6 \%$ de los casos el cadáver fue hallado en el mar y en el $47,4 \%$ en agua dulce. La estación del año en que hubo más fallecimientos fue el verano (31 casos), seguida de la primavera (21 casos).

En relación con los hallazgos macroscópicos apreciados por el patólogo forense durante la autopsia, había líquido de sumersión en el estómago en el $57 \%$ de los casos, pero sólo en el $12,8 \%$ el volumen cuantificado fue superior a $500 \mathrm{ml}$. El corazón tenía un peso superior a 500 g en 15 varones y sólo en tres mujeres. Como hallazgos macroscópicos, se apreció hongo de espuma en el $62 \%$ de las víctimas, edema pulmonar en el 95\%, equimosis de Paltauf en el $14 \%$, espuma traqueobronquial en el $75 \%$ y derrame pleural en el $8 \%$. 
El $82 \%$ (64 casos) de las víctimas presentaba un peso de los pulmones superior a $1.000 \mathrm{~g}$ y el $18 \%$ (14 casos) un peso inferior a $1.000 \mathrm{~g}$ (Tabla 1 ). Respecto al peso pulmonar, encontramos diferencias significativas entre ambos sexos $(p<0,0001)$ : frente al $45,5 \%$ de las mujeres, sólo un $7,1 \%$ de los hombres tenían un peso pulmonar menor de $1.000 \mathrm{~g}$. Los cadáveres rescatados del mar presentaban un peso pulmonar mayor que los procedentes de agua dulce ( $p=0,0008$ ) (Tabla 2); dentro de este grupo, en los varones se halló un aumento significativo del peso pulmonar respecto de los ahogados en agua dulce ( $p=0,046)$, lo cual no pudo confirmarse en las mujeres.

El mayor peso de los pulmones se relaciona significativamente con el hallazgo necrópsico de hongo de espuma y con la presencia de espuma traqueobronquial, pero sólo en el caso de los hombres.

Entre aquellos en que el peso pulmonar fue inferior a $1.000 \mathrm{~g}$ ( 14 casos), sólo tres (3,8\% del total) presentaron las siguientes características diferenciales:

- Pulmones sin patología macroscópica.

- Ausencia de hallazgos necrópsicos propios de la sumersión, como el hongo de espuma o espuma traqueobronquial.

- Ausencia de otra causa de muerte violenta.

En estos tres casos, el estudio histopatológico del pulmón reveló la presencia de rotura de septos alveolares y de edema alveolar (hallazgos inespecíficos, pero que se observan en víctimas de sumersión); además, se constató la presencia de alteraciones coronarias no graves en el estudio necrópsico, aunque carecían de antecedentes médicos de interés. Las pruebas de biología encontraron la misma especie de diatomeas (Navicula, Synedra) en la médula ósea y el bazo. Estos tres casos podrían corresponderse con los denominados pulmones secos o dry-drowning.

En el estudio químico-toxicológico se detectaron psicofármacos, etanol y drogas de abuso: en 28 casos (36\%) etanol en sangre, benzodiacepinas (16), antidepresivos (10), cocaína (3), opiáceos (3), cannabis (1) y otros (3) (Tabla 3). En 12 casos (15\%) se detectó etanol junto a otra droga, siendo la combinación más frecuente $(6,4 \%)$ con antidepresivos. En el $18 \%$ de los ahogados se detectó más de una droga, siendo la combinación más frecuente antidepresivos y benzodiacepinas (11,5\%). El 30\% de los hombres ahogados presentaban una cifra de alcohol en sangre $>0,3 \mathrm{~g} / \mathrm{l}$, mientras que en las mujeres la frecuencia era del $18 \%$. Nuestros resultados muestran una tendencia, no significativa, entre el mayor peso de los pulmones y la presencia de etanol en sangre; el peso de los pulmones era aún mayor cuando además se encontraban otras drogas en el cadáver, como benzodiacepinas, opiáceos y cannabis.

\section{Discusión}

En este artículo se describen 78 casos de muertes por sumersión ocurridos en la provincia de Málaga entre los años 2004 y 2011. Se analizaron los factores individuales y circunstanciales, los hallaz-

Tabla 1.

Peso de los pulmones en muertos por sumersión.

\begin{tabular}{|c|c|c|c|c|c|c|}
\hline & \multicolumn{2}{|c|}{ No verificado } & \multicolumn{2}{|c|}{ Verificado } & \multicolumn{2}{|c|}{ Total } \\
\hline & $\mathbf{N}$ & $\%$ & $\mathbf{N}$ & $\%$ & $\mathbf{N}$ & $\%$ \\
\hline \multicolumn{7}{|l|}{ Peso pulmonar } \\
\hline$<1.000 \mathrm{~g}$ & 13 & 23,6 & 1 & 4,3 & 14 & 17,9 \\
\hline$\geq 1.000 \mathrm{~g}$ & 42 & 76,4 & 22 & 95,7 & 64 & 82,1 \\
\hline Total & 55 & 100 & 23 & 100 & 78 & 100 \\
\hline \multicolumn{7}{|l|}{ Hombre } \\
\hline$<1.000 \mathrm{~g}$ & 3 & 8,3 & 1 & 5 & 4 & 7,1 \\
\hline$\geq 1.000 \mathrm{~g}$ & 33 & 91,7 & 19 & 95 & 52 & 92,9 \\
\hline Total & 36 & 100 & 20 & 100 & 56 & 100 \\
\hline \multicolumn{7}{|l|}{ Mujer } \\
\hline$<1.000 \mathrm{~g}$ & 10 & 52,6 & 0 & 0 & 10 & 45,4 \\
\hline$\geq 1.000 \mathrm{~g}$ & 9 & 47,4 & 3 & 100 & 12 & 54,6 \\
\hline Total & 19 & 100 & 3 & 100 & 22 & 100 \\
\hline
\end{tabular}




\begin{tabular}{|c|c|c|c|c|c|c|}
\hline & \multicolumn{2}{|c|}{ Agua salada } & \multicolumn{2}{|c|}{ Agua dulce } & \multicolumn{2}{|c|}{ Total } \\
\hline & $\mathbf{N}$ & $\%$ & $\mathbf{N}$ & $\%$ & $\mathbf{N}$ & $\%$ \\
\hline \multicolumn{7}{|l|}{ Peso pulmonar } \\
\hline$<1.000 \mathrm{~g}$ & 3 & 7,3 & 11 & 29,7 & 14 & 17,9 \\
\hline$\geq 1.000 \mathrm{~g}$ & 38 & 92,7 & 26 & 70,3 & 64 & 82,1 \\
\hline Total & 41 & 100 & 37 & 100 & 78 & 100 \\
\hline \multicolumn{7}{|l|}{ Hombre } \\
\hline$<1.000 \mathrm{~g}$ & 1 & 2,8 & 3 & 15 & 4 & 7,1 \\
\hline$\geq 1.000 \mathrm{~g}$ & 35 & 97,2 & 17 & 85 & 52 & 92,9 \\
\hline Total & 36 & 100 & 20 & 100 & 56 & 100 \\
\hline \multicolumn{7}{|l|}{ Mujer } \\
\hline$<1.000 \mathrm{~g}$ & 2 & 40 & 8 & 47 & 10 & 45,5 \\
\hline$\geq 1.000 \mathrm{~g}$ & 3 & 60 & 9 & 53 & 12 & 54,5 \\
\hline Total & 5 & 100 & 7 & 100 & 22 & 100 \\
\hline
\end{tabular}

\begin{tabular}{ccccccc}
\hline Etanol & Cocaína & Opiáceos & Cannabis & Benzodiacepinas & Antidepresivos & Otros \\
\hline 28 & 3 & 3 & 1 & 16 & 10 & 3 \\
\hline
\end{tabular}

Tabla 3.

Tóxicos en sangre de cadáveres recuperados del agua. gos macroscópicos y las pruebas complementarias realizadas. Detectamos tres casos diagnosticados por el Servicio de Patología Forense del IMLMA de muerte por sumersión con un peso pulmonar inferior a $1.000 \mathrm{~g}$, ausencia de hallazgos macroscópicos indicativos de entrada del medio de sumersión en las vías respiratorias y de otra causa que pudiera explicar el fallecimiento; sin embargo, el estudio histopatológico mostró hallazgos propios de la sumersión, si bien no específicos de ella, como son la rotura de los tabiques alveolares y la presencia de edema alveolar; también se detectaron diatomeas en las pruebas de biología realizadas.

Al igual que en otros estudios ${ }^{7}$, la etiología médicolegal accidental fue la más frecuente en nuestro estudio, con un $69 \%$, seguida de la suicida con un $28 \%$ y en último lugar la homicida con un $3 \%$.

El peso de los pulmones depende del sexo y de la edad del sujeto: es mayor en los hombres que en las mujeres y en los jóvenes que en la senectud. El volumen del pulmón está directamente relacionado con la constitución física del individuo, la capacidad vital pulmonar y el gasto cardíaco en combinación con el tiempo de agonía ${ }^{14}$. Otros factores a considerar en relación con el peso de los pulmones son la cantidad de agua aspirada y el tiempo de supervivencia. Cabe esperar que los sujetos jóvenes y con mayor tiempo de supervivencia aspiraran mayor cantidad del medio de sumersión y, por tanto, el peso pulmonar sería más elevado.

En nuestro estudio, los pulmones de mayor peso están relacionados, aunque no de manera estadísticamente significativa, con la sumersión en agua salada, lo cual es concordante con la bibliografía existente. Algunos autores concluyen que la diferencia entre ahogados en agua con mayor salinidad respecto a los de menor es la presencia de mayor derrame pleural en los primeros ${ }^{15}$.

En contraste con el estudio realizado por Zhu et al. ${ }^{14}$, que describen una disminución del peso pulmonar de manera gradual dependiendo del intervalo post mórtem, en nuestro trabajo no existe tal correlación. Esta discordancia puede ser explicada por el intervalo post mórtem considerado, que en nuestro estudio era de hasta 6 días y en el trabajo de Zhu era de 8 días.
Tabla 2.

Peso de los pulmones según la salinidad del agua. 
En los tratados de medicina legal se cita generalmente que un $10-15 \%$ de los cadáveres recuperados del agua carecían de líquido en sus pulmones (pulmones secos o dry-drowning). Se han alegado diversos mecanismos para poder dar explicación a tal hallazgo, como un laringoespasmo reflejo causado por la entrada de líquido ${ }^{6}$ o una inhibición cardíaca refleja por estimulación del vago o por choque térmico. Según Lunetta et al. ${ }^{16}$, el volumen de líquido aspirado varía de unas víctimas a otras y depende de factores como la frecuencia y la duración del laringoespasmo, el número y la profundidad de los movimientos respiratorios realizados antes de la muerte, y el tiempo transcurrido antes de la parada cardíaca. Estos mismos autores sugieren en su estudio que la incidencia real de los pulmones secos es más baja que la estimada clásicamente, siendo tan sólo del $2 \%$. Además, estiman que los cadáveres encontrados en el agua con pulmones aparentemente normales podrían encubrir más casos de muertes naturales en el agua de los diagnosticados habitualmente.

En nuestro estudio hemos encontrado tres casos $(3,8 \%)$ de cadáveres retirados del agua con pulmones aparentemente normales y con algunos hallazgos histopatológicos inespecíficos, como son la rotura de tabiques alveolares y edema alveolar. En estos casos analizamos las relaciones peso pulmón/peso corazón y peso pulmón/peso general, que ayudan al diagnóstico de muerte por sumersión ${ }^{17}$. Los resultados obtenidos se encontraban por debajo de los límites establecidos como propios de la muerte por sumersión $(<19,5 \mathrm{~g} / \mathrm{kg}$ ). Estos hallazgos sugieren que estos tres casos no se correspondían con las características de peso propias de los ahogados. Por tanto, podíamos encontrarnos ante sujetos que fallecen en el agua por causas naturales, como arritmias cardíacas (cardiopatías sin alteración estructural). En este sentido, Lunetta et al. ${ }^{18}$, en su estudio sobre 165 cadáveres encontrados en el agua, comprobaron una mutación del gen LQT2 en el cadáver de una mujer cuya muerte había sido etiquetada inicialmente de etiología suicida.

Estos tres casos detectados en nuestro estudio serían candidatos a una autopsia molecular para descartar la presencia de algún tipo de canalopatía que pudiera explicar el mecanismo de la muerte. Por ello, consideramos que el patólogo forense debe utilizar todas las herramientas disponibles a su alcance para intentar arrojar un poco de luz en la investigación médico-legal de las muertes por sumersión.

\section{Conclusiones}

Nuestro estudio indica una menor incidencia de pulmones secos respecto a las cifras admitidas clásicamente, así como la dificultad diagnóstica de la muerte por sumersión, sin que exista, hasta el momento, hallazgo alguno específico de este tipo de muertes. En estos casos de pulmones secos estaría indicado realizar un estudio molecular.

Los autores declaran no tener ningún conflicto de intereses.

\section{Bibliografía}

1. Simonin C. Sumersión. En: Medicina legal judicial. Barcelona: JIMS;1962. p. 225-43.

2. Idris A, Berg R, Bierens J, et al. ILCOR Advisory Statement: recommended guidelines for uniform reporting of data from browning. The "Utstein Style". Circulation. 2003; 108:2565-74.

3. World Health Organization. Fact sheet on drowning Fact sheet. 2010;347. November. Disponible en: www.who.int/violence prev.

4. Bell MD. Drowning. En: Dolinak D, Matshes E, Lew E, editores. Forensic pathology. Principles and practice. Amsterdam: Elsevier Academic Press; 2005. p. 227-35.
5. Instituto Nacional de Estadística. Disponible en: www. ine.es

6. Kinght B. Immersion deaths. En: Knight B, editor Forensic pathology. London: Edward Arnolds; 1991.

7. Romero Palanco JL. Muertes por sumersión. Revisión y actualización de un tema clásico de la medicina forense. Cuad Med Forense. 2007;13(48-49):99-130.

8. Shkrum MJ, Ramsay DA. Forensic pathology of trauma. Totowa: Humana Press; 2007. p. 243-93.

9. Modell JH, Bellefleur M, Davis JH. Drowning without aspiration: is this an appropriate diagnosis? J Forensic Sci. 1999;44:1119-23. 
10. Modell JH, Moya F. Effects of volume of aspirated fluid during chlorinated fresh water drowning. Anesthesiology. 1966; 27:662-72.

11. Vallejo G, Azparren JE, Sánchez de León S, et al. Pruebas biológicas complementarias en las muertes por sumersión. Rev Esp Med Legal. 2012;38(1):17-27.

12. Orlowski JP, SpizIman D. Drowning: rescue, resuscitation and reanimation. Pediatr Clin North Am. 2001;48:627-46.

13. Recomendación No 99(3) del Consejo de Ministros de los estados miembros para la armonización metodológica de las autopsias médico-legales. Rev Esp Med Leg. 1999; XXIII(86-87).

14. Zhu BL, Quan L, Li DR, et al. Postmortem lung weight in drownings: a comparison with acute asphyxiation and cardiac death. Leg Med. 2003;5:20-6.
15. Spitz WU, Spitz DJ, editores. Spitz and Fisher's Medicolegal investigation of death: guidelines for the application of pathology to crime investigation. 4th ed. Springfield, IL: Charles C. Thomas; 2006. p. 849.

16. Lunetta P, Modell JH, Sajantila A. What is the incidence and significance of "dry-lungs" in bodies found in water? Am J Forensic Med Pathol. 2004;25:291-301.

17. Azparren JE, Cubero C, Perucha E, et al. Comparison between lung weight and blood strontium in bodies found in seawater. Forensic Sci Int. 2007;168:12832.

18. Lunetta $P$, Levo A, Männikkö A, et al. Death in bathtub revised with molecular genetics: a victim with suicidal traits and a LQTS gene mutation. Forensic Sci Int. 2002;130:122-4. 